\title{
Niedrigrisiko-Prostatakarzinom: Therapie und gesundheitsbezogene Lebensqualität
}

\begin{abstract}
Die radikale Prostatektomie bereits im Frühstadium der Erkrankung verspricht hohe Heilungsraten, erhöht aber die therapiebedingte Morbidität. Experten empfehlen für Niedrigrisiko-Patienten die aktive Überwachung.
\end{abstract}

Um Patienten mit einem organbegrenzten Prostatakarzinom $(\mathrm{PCa})$ und niedrigem Progressrisiko eine etwaige chirurgische Überbehandlung zu ersparen, empfehlen aktuelle Leitlinien die aktive

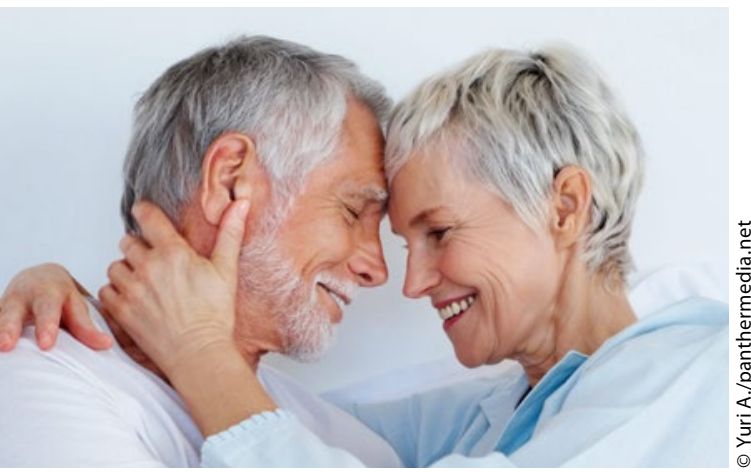

Aktive Überwachung beeinträchtigte die Sexualfunktion deutlich weniger als eine radikale Prostatektomie.
Überwachung (AS) als erste Behandlungsstrategie. Studien bescheinigtem diesem Ansatz adäquate, mit denen einer definitiven Therapie vergleichbare onkologische Ergebnisse. Aspekte der gesundheitsbezogenen Lebensqualität (HRQoL) sind daher bei der Therapieentscheidung wichtig. Für aktiv überwachte Niedrigrisiko-Patienten verschlechtert sich die physische HRQoL zwar nicht in dem Ausmaß wie für definitiv Behandelte. Demgegenüber können Ängste, die potenziell lebensrettende Therapie nicht erhalten zu haben, die mentale Gesundheit negativ beeinflussen. In ihrer Studie verglichen die Forscher prospektiv, mittels validierter Fragebögen HRQoL-Daten von Patienten mit Niedrigrisiko-PCa (klinisches Tumorstadium T1-T2a, Gleasonscore der Prostatabiopsien $\leq 6$ und PSA-Spiegel $<10 \mathrm{ng} / \mathrm{ml}$ ), die entweder radikal ope- riert oder aktiv überwacht worden waren. Von den 389 Patienten unterzogen sich $59 \%$ einer radikalen Prostatektomie (RP). Die Patienten im AS-Arm (20\%) erhielten im ersten Jahr nach Diagnosestellung keine definitive Therapie und entweder den Eintrag „AS als Behandlungsstrategie" in die Krankenakte oder mindestens einen PSA-Test bzw. wiederholte Biopsien binnen 18 Monaten.

Die RP führte zu allen Zeitpunkten zu signifikanten Einbußen der Sexual- und Blasenfunktion im Vergleich zur ASGruppe. Die Unterschiede zwischen beiden Gruppen hinsichtlich von Aspekten der geistigen Gesundheit waren nach einem Jahr statistisch nicht signifikant.

Fazit: Die aktive Überwachung verschlechtert nach drei Jahren nicht die geistige Gesundheit von Patienten mit Niedrigrisiko-Prostatakarzinom im Vergleich zur radikalen Prostatektomie. Erwartungsgemäß führt die chirurgische Option zu ungünstigeren HRQoLErgebnisse in den Punkten Blasen- und Sexualfunktion. Wolfgang Zimmermann

Jeldres C et al. Prospective Quality-of-Life outcomes for low-risk prostate cancer: active surveillance versus radical prostatectomy. Cancer. 2015;121(14):2465-73.

\section{Hochdosis-Radiotherapie des Prostatakarzinoms: Symptombeurteilung aus Patientensicht}

\author{
Eine hochdosierte Radiotherapie (79 Gy und höher) verbessert bei Patienten \\ mit Prostatakrebs das biochemische krankheitsfreie Überleben. Jetzt liegt \\ eine Analyse der patientenberichteten Ergebnisse zu 3-D-CRT und IMRT vor.
}

$\mathrm{D}$ ie Verabreichung der Hochdosis am Zielort per intensitätsmodulierter Strahlentherapie (IMRT) scheint das umgebende gesunde Gewebe effektiver zu schonen als die dreidimensionale konformale Bestrahlung (3-D-CRT). Das Team um Deborah W. Bruner verglich erstmals die Symptomeinschätzung von Patienten nach IMRT oder 3-D-CRT bezüglich therapieassoziierter Schäden an Blase, Darm bzw. erektiler Funktion. Die Patienten gehörten dem Hochdosis-Arm der Phase-III-Studie 0126 der Radiation Therapy Oncology Group an. Darin wur- den hoch und konventionell dosierte Bestrahlung bei Patienten mit Prostatakarzinom mittleren Risikos gegenübergestellt. Patienten in der 3-D-CRT-Gruppe des Hochdosis-Arms erhielten fraktioniert 55,8 Gy auf Prostata und proximale Samenblasen, gefolgt von auf die Prostata begrenzten 23,4 Gy. Mit IMRT Behandelte bekamen in Fraktionen ebenfalls 79,2 Gy auf Prostata plus proximale Samenblasen. Patientenberichtete Ergebnisse anhand etablierter Scores zur subjektiven Beurteilung von Blasen-, Darmund sexuellen Funktionen ermittelten die Forscher zu den Zeitpunkten 0, 3, 6, 12 und 24 Monate. Für sie unerwartet ergab die vorläufige Datenauswertung zu keiner Zeit signifikante Unterschiede der individuellen Symptombeurteilung bezüglich der drei therapieassoziierten Funktionen.

Fazit: Trotz geringerer Belastung gesunden Gewebes mit IMRT im Rahmen einer Radiotherapie des Prostatakarzinoms demonstrierte dieser Vergleich zwischen IMRT und 3-D-CRT in identischer Dosierung keine Unterschiede in den patientenberichteten Ergebnissen zu Blasen-, Darm- oder erektiler Funktion.

Wolfgang Zimmermann

Bruner DW et al. Preliminary patient-reported outcomes analysis of 3-dimensional radiation therapy versus intensity-modulated radiation therapy on the high-dose arm of the Radiation Therapy Oncology Group (RTOG) 0126 prostate cancer trial. Cancer. 2015;121(14):2422-30. 